INSTITUT NATIONAL DE RECHERCHE EN INFORMATIQUE ET EN AUTOMATIQUE

\title{
Lipschitz stability for the inverse Robin problem
}

\author{
Eva SINCICH
}

$\mathbf{N}^{\circ} 6103$

Octobre 2006

Thème NUM

\section{apport}

de recherche 



\title{
Lipschitz stability for the inverse Robin problem
}

\author{
Eva SINCICH * \\ Thème NUM — Systèmes numériques \\ Projets Apics \\ Rapport de recherche $\mathrm{n}^{\circ} 6103$ - Octobre 2006 - 25 pages
}

\begin{abstract}
We are concerned with an inverse problem arising in corrosion detection. We prove a Lipschitz stability estimate for a scalar Robin coefficient on an inaccessible portion of the boundary by electrostatic measurements performed on the accessible one.
\end{abstract}

Key-words: Robin problem, inverse boundary problem, corrosion.

* INRIA, BP 93, 06902 Sophia-Antipolis Cedex, France, esincich@sophia.inria.fr 


\section{Stabilité Lipschitzienne pour le problème inverse de Robin}

Résumé : On considère un problème inverse lié a la détection de la corrosion. On prouve un résultat de stabilité Lipschitz pour un coefficient de Robin scalaire sur une partie inaccessible de la frontière depuis des mesures électrostatiques disponibles sur la partie accessible.

Mots-clés : Problème de Robin, problème inverse à la frontière, corrosion. 


\section{Introduction}

In the following we shall consider the inverse problem of recovering a scalar Robin coefficient. The direct problem associated with the inverse one, is the following

$$
\begin{cases}\Delta u=0, & \text { in } \Omega, \\ \frac{\partial u}{\partial \nu}=g, & \text { on } \Gamma_{2}, \\ \frac{\partial u}{\partial \nu}=-L u, & \text { on } \Gamma_{1}, \\ u=0, & \text { on } \Gamma_{D},\end{cases}
$$

where $\Gamma_{1}$ and $\Gamma_{2}$ are two open, disjoint portions of $\partial \Omega$ such that $\Gamma_{D}=\partial \Omega \backslash\left(\overline{\Gamma_{1} \cup \Gamma_{2}}\right)$.

According to this model, $\Omega$ represents the electrostatic conductor, $u$ is the harmonic potential, $g$ is the prescribed current density on the accessible part of the boundary $\Gamma_{2}$, whereas on $\Gamma_{1}$, the portion which is out of reach, we prescribe a Robin boundary condition with a scalar Robin coefficient $L$.

The aim of the inverse problem that we shall study is to recover the scalar Robin coefficient $L$ when a fixed choice of the current density $g$ and the measurement of the corresponding boundary voltage $\left.u\right|_{\Gamma_{2}}$ on $\Gamma_{2}$ are available.

In the following we shall treat the stability issue for this inverse problem. Such an issue has been already discussed by Chaabane and Jaoua in [15]. The authors provided an identifiability result as well as a Lipschitz stability estimate à la Bellout and Friedman for a Robin coefficient which depends on a scalar parameter only. The new feature of the present work consists in a quantitative evaluation of such a Lipschitz rate of stability, which is no longer a directional stability as in [15] and which is obtained with a constructive approach.

Moreover, Alessandrini, Del Piero, Rondi [5] and Chaabane, Fellah, Jaoua, Leblond [13] have established a logarithmic stability result for a non necessarily constant Robin coefficient, namely of the type

$$
L=L(x), x \in \Gamma_{1} .
$$

In [14], Chaabane, Jaoua and Leblond have provided a constructive procedure in order to solve the Robin problem.

We refer also to Fasino and Inglese [16, 17, 18], who have introduced numerical methods relied on the thin-plate approximation and the Galerkin method.

In the corrosion literature we can find another type of boundary condition which postulates a nonlinear relationship between the potential and the normal current density and it is associated to the name of Butler and Volmer.

Such type of problem has been studied by Brian, Kavian, Vogelius and Xu [12, 20, 26]. The authors have dealt with the uniqueness and the existence issues for this problem with a special choice of the nonlinearity, that is of the type

$$
\frac{\partial u}{\partial \nu}=\lambda(\exp (\alpha u)-\exp (-(1-\alpha) u)) .
$$

RR $n^{\circ} 6103$ 
Moreover, in $[7,8]$ Alessandrini and the author have discussed the same problem but with a more general choice of the nonlinear profile of the form

$$
\frac{\partial u}{\partial \nu}=f(u),
$$

establishing a logarithmic stability estimate as well as a reconstruction result.

The present work fits in the framework of the study of the stability for coefficients which depend on a finite number of parameters only. Such an issue has been developed by many authors, among them we cite Alessandrini, Bacchelli, Beretta, Chaabane, Jaoua, Vessella $[4,9,10,15]$. Indeed, for what concerns the present problem, in [5] it has been shown in a 2-dimensional setting the stability for a functional Robin coefficient is at best logarithmic. Unfortunately this kind of stability is inconvenient for the application. For this reason is usefull to introduce some reasonable simplifications which are phisically meaningfull (as remarked in [9]) and that lead to a better stability, namely of Lipschitz type.

\section{Main assumptions and results}

\section{A priori information on the domain}

We shall assume throughout that $\Omega$ is a bounded, connected domain in $\mathbb{R}^{n}, n \geqslant 2$ such that $\operatorname{diam}(\Omega) \leqslant D$ and with Lipschitz boundary $\partial \Omega$ with constants $r_{0}, M$. Moreover, we assume that the portions of the boundary $\Gamma_{i}$ are contained respectively into surfaces $S_{i}$, $i=1,2$ which are $C^{1, \alpha}$ smooth with constants $r_{0}, M$.

We also suppose that the boundary of $\Gamma_{i}$, within $S_{i}$, is of $C^{1, \alpha}$ class with constants $r_{0}, M$. We introduce some notation that we shall use in the sequel, for every $\rho>0$ and $i=1,2$, we set

$$
\begin{gathered}
U_{\rho}^{i}=\left\{x \in \bar{\Omega}: \operatorname{dist}\left(x, \partial \Omega \backslash \Gamma_{i}\right)>\rho\right\}, \\
\Gamma_{i, \rho}=U_{\rho}^{1} \cap \Gamma_{i} .
\end{gathered}
$$

In some places, it will be necessary to isolate one privileged coordinate direction, to this purpose, we shall use the following notation for a point $x \in \mathbb{R}^{n}, x=\left(x^{\prime}, x_{n}\right)$, with $x^{\prime} \in$ $\mathbb{R}^{n-1}, x_{n} \in \mathbb{R}$.

\section{A priori information on the boundary data}

The current flux $g$ is a prescribed function such that

$$
\begin{gathered}
\|g\|_{C^{0, \alpha}\left(\Gamma_{2}\right)} \leqslant G, \\
\|g\|_{L^{\infty}\left(\Gamma_{2,2 r_{0}}\right)} \geqslant m>0 .
\end{gathered}
$$




\section{A priori information on the scalar Robin coefficient}

Given $\Lambda>0$, we assume that the scalar Robin coefficient $L$ satisfies

$$
0<L \leqslant \Lambda .
$$

From now on we shall refer to the a priori data as to the set of quantities $r_{0}, M, \alpha, \Lambda, G, D, m$. In the sequel we shall denote by $\eta(t)$ and $\omega(t)$, two positive increasing functions defined on $(0,+\infty)$, that satisfy

$$
\begin{gathered}
\eta(t) \geqslant \exp \left[-\left(\frac{t}{c}\right)^{-\gamma}\right], \quad \text { for every } 0<t \leqslant G, \\
\omega(t) \leqslant C|\log (t)|^{-\theta}, \quad \text { for every } 0<t<1,
\end{gathered}
$$

where $c>0, C>0, \gamma>1,0<\theta<1$ are constants depending on the a priori data only. The statement of the main result is the following.

Theorem 2.1. There exists a constant $C>0$, depending on the a priori data only, such that

$$
\left|L_{0}-L_{1}\right| \leqslant C\left\|u_{0}-u_{1}\right\|_{L^{2}\left(\Gamma_{2}\right)},
$$

where $u_{i}, i=0,1$ is the solution to (1.1) with $L=L_{i}$.

\section{$3 \quad$ Lipschitz stability}

Given $L_{0}, L_{1}>0$ and $t \in[0,1]$, we shall denote by $L_{t}$ the following

$$
L_{t}=-\left[L_{0}+t\left(L_{1}-L_{0}\right)\right] .
$$

Moreover, let $u_{t} \in H^{1}(\Omega)$ be the weak solution to

$$
\begin{cases}\Delta u_{t}=0, & \text { in } \Omega, \\ \frac{\partial u_{t}}{\partial \nu}=g, & \text { on } \Gamma_{2}, \\ \frac{\partial u_{t}}{\partial \nu}=-L_{t} u_{t}, & \text { on } \Gamma_{1}, \\ u_{t}=0, & \text { on } \Gamma_{D} .\end{cases}
$$

As a weak solution to the problem (3.2) we shall mean a function $u_{t} \in H^{1}(\Omega)$, such that $u_{t}=0$ which satisfies

$$
\int_{\Omega} \nabla u_{t} \nabla \rho=\int_{\Gamma_{2}} g \rho-\int_{\Gamma_{1}} L_{t} u_{t} \rho
$$

for every $\rho \in H^{1}(\Omega)$ such that $\rho=0$ on $\Gamma_{D}$.

The existence and the uniqueness of the solution to (3.2) is a consequence of standard theory on boundary value problem. 
Proposition 3.1. There exists a constant $C>0$ depending on the a priori data only such that

$$
\int_{\Omega}\left|\nabla u_{t}\right|^{2} \leqslant C
$$

for each $t \in[0,1]$.

Proof. Let us choose $\rho=u_{t}$, then by (3.3) we have that

$$
\int_{\Omega}\left|\nabla u_{t}\right|^{2}=\int_{\Gamma_{2}} g u_{t}-\int_{\Gamma_{1}} L_{t} u_{t}^{2}
$$

From which follows

$$
\int_{\Omega}\left|\nabla u_{t}\right|^{2} \leqslant \int_{\Gamma_{2}} g u_{t}
$$

Let $\varepsilon>0$, by the Schwartz inequality we have that

$$
\int_{\Omega}\left|\nabla u_{t}\right|^{2} \leqslant \frac{1}{\varepsilon} \int_{\Gamma_{2}} g^{2}+\varepsilon \int_{\Gamma_{2}}\left|u_{t}\right|^{2} .
$$

By a standard trace inequality (see for instance [1]) we have that there exists a constant $C>0$ depending on the a priori data only such that

$$
\int_{\Omega}\left|\nabla u_{t}\right|^{2} \leqslant \frac{1}{\varepsilon} \int_{\Gamma_{2}} g^{2}+C \varepsilon \int_{\Gamma_{2}}\left|\nabla u_{t}\right|^{2}
$$

Choosing $\varepsilon=\frac{1}{2 C}$ we have that

$$
\int_{\Omega}\left|\nabla u_{t}\right|^{2} \leqslant \frac{1}{C} \int_{\Gamma_{2}} g^{2} .
$$

Hence by (2.3) the thesis follows.

Theorem 3.2. Let $t \in[0,1]$ and let $u_{t} \in H^{1}(\Omega)$ be the solution to (3.2), there exists a constant $C>0$ depending on the a priori data only such that

$$
\left\|u_{t}\right\|_{C^{0, \alpha}\left(\Gamma_{1}\right)} \leqslant C .
$$

Moreover, for any $\rho \in\left(0, r_{0}\right), u_{t} \in C^{1, \alpha}\left(U_{\rho}^{1}\right)$ and there exists a constant $C_{\rho}>0$ depending on the a priori data and on $\rho$ only, such that the following estimate holds

$$
\left\|u_{t}\right\|_{C^{1, \alpha}\left(U_{\rho}^{1}\right)} \leqslant C_{\rho} E,
$$

where $0<\alpha<1$ is a constant depending on $r_{0}, M, n$ only. 
Proof. For any $z_{0} \in \Gamma_{1}$ and for any $\rho>0$, we shall denote

$$
\begin{aligned}
\Gamma_{\rho}\left(z_{0}\right) & =\Omega \cap B_{\rho}\left(z_{0}\right), \\
\Delta_{\rho}\left(z_{0}\right) & =\bar{\Gamma}_{\rho}\left(z_{0}\right) \cap \partial \Omega .
\end{aligned}
$$

Let $0<\rho_{1}<\rho_{2} \leqslant r_{0}$ and let us consider a test function $\varphi \in \mathrm{C}^{1}(\bar{\Omega})$ such that

i) $0 \leqslant \varphi \leqslant 1$

ii) $\varphi=1$ in $\Gamma_{\rho_{1}}\left(z_{0}\right)$ and $\varphi=0$ in $\Omega \backslash \Gamma_{\rho_{2}}\left(z_{0}\right)$;

iii) $|\nabla \varphi| \leqslant \frac{2}{\rho_{2}-\rho_{1}}$.

For a sake of brevity, in the following we shall replace $u_{t}$ with $u$ and $L_{t}$ with $L$.

For any integer $s \geqslant 2$, let us define the function $\psi=|u|^{s-2} u \varphi^{2}$. Hence, choosing $\psi$ as test function in the weak formulation of the problem (3.3) we have that

$$
\begin{aligned}
& \int_{\Gamma_{\rho_{2}}\left(z_{0}\right)}(s-1)|\nabla u|^{2}|u|^{s-2} \varphi^{2}+\int_{\Gamma_{\rho_{2}}\left(z_{0}\right)} 2 \nabla u \cdot \nabla \varphi|u|^{s-2} u \varphi= \\
& \int_{\Delta_{\rho_{2}}\left(z_{0}\right)} L|u|^{s} \varphi^{2} .
\end{aligned}
$$

Hence,

$$
\begin{aligned}
\int_{\Gamma_{\rho_{2}}\left(z_{0}\right)}(s-1)|\nabla u|^{2}|u|^{s-2} \varphi^{2} & \leqslant\left.\left|\int_{\Gamma_{\rho_{2}}\left(z_{0}\right)} 2 \nabla u \cdot \nabla \varphi\right| u\right|^{s-2} u \varphi \mid+ \\
& +\left.\left|\int_{\Delta_{\rho_{2}}\left(z_{0}\right)} L\right| u\right|^{s} \varphi^{2} \mid .
\end{aligned}
$$

By applying the Hölder inequality to the first term on the right hand side of (3.15), we obtain

$$
\left.\left|\int_{\Gamma_{\rho_{2}}\left(z_{0}\right)} 2 \nabla u \cdot \nabla \varphi\right| u\right|^{s-2} u \varphi \mid \leqslant \frac{4}{\rho_{2}-\rho_{1}}\left(\int_{\Gamma_{\rho_{2}}\left(z_{0}\right)}|\nabla u|^{2}|u|^{s-2} \varphi^{2}\right)^{\frac{1}{2}}\left(\int_{\Gamma_{\rho_{2}}\left(z_{0}\right)}|u|^{s}\right)^{\frac{1}{2}}
$$

By the Schwartz inequality, it then follows that for every $\varepsilon>0$

$$
\begin{aligned}
& \left.\left|\int_{\Gamma_{\rho_{2}}\left(z_{0}\right)} 2 \nabla u \cdot \nabla \varphi\right| u\right|^{s-2} u \varphi \mid \leqslant \\
& \leqslant \varepsilon\left(\int_{\Gamma_{\rho_{2}}\left(z_{0}\right)}|\nabla u|^{2}|u|^{s-2} \varphi^{2}\right)+\frac{16}{\left(\rho_{2}-\rho_{1}\right)^{2} \varepsilon}\left(\int_{\Gamma_{\rho_{2}}\left(z_{0}\right)}|u|^{s}\right)
\end{aligned}
$$


Let us now consider the second term on the right hand side of (3.15). Furthermore by a trace inequality, see for instance [1, Theorem 5.22], we infer that

$$
\left.\left|\int_{\Delta_{\rho_{2}}\left(z_{0}\right)} L\right| u\right|^{s} \varphi^{2}\left|\leqslant C L \int_{\Gamma_{\rho_{2}}\left(z_{0}\right)}\right| \nabla\left(|u|^{s} \varphi^{2}\right) \mid
$$

where $C>0$ is a constant depending on the a priori data only. Hence by the Schwartz inequality, it then follows that for every $\varepsilon>0$

$$
\begin{aligned}
& \left.\left|\int_{\Delta_{\rho_{2}}\left(z_{0}\right)} L\right| u\right|^{s} \varphi^{2} \mid \leqslant \\
& \leqslant \varepsilon \int_{\Gamma_{\rho_{2}}\left(z_{0}\right)}|u|^{s-2}|\nabla u|^{2} \varphi^{2}+\frac{s^{2} C^{2} L^{2}}{\varepsilon} \int_{\Gamma_{\rho_{2}}\left(z_{0}\right)}|u|^{s}+\frac{4 C L}{\rho_{2}-\rho_{1}} \int_{\Gamma_{\rho_{2}}\left(z_{0}\right)}|u|^{s}
\end{aligned}
$$

Inserting (3.16) and (3.18) in (3.15), we obtain

$$
\begin{aligned}
& (1-2 \varepsilon)\left(\int_{\Gamma_{\rho_{2}}\left(z_{0}\right)}|u|^{s-2}|\nabla u|^{2} \varphi^{2}\right) \leqslant \\
\leqslant & \left(\frac{16}{\left(\rho_{2}-\rho_{1}\right)^{2} \varepsilon}+\frac{L^{2} s^{2} C^{2}}{\varepsilon}+\frac{4 C L}{\rho_{2}-\rho_{1}}\right)\left(\int_{\Gamma_{\rho_{2}}\left(z_{0}\right)}|u|^{s}\right)
\end{aligned}
$$

Choosing $\varepsilon=\frac{1}{4}$ in the above inequality we have that

$$
\int_{\Gamma_{\rho_{1}}\left(z_{0}\right)}|u|^{s-2}|\nabla u|^{2} \leqslant\left(\frac{32}{\left(\rho_{2}-\rho_{1}\right)^{2} \varepsilon}+\frac{2 L^{2} s^{2} C^{2}}{\varepsilon}+\frac{2 C L}{\rho_{2}-\rho_{1}}\right)\left(\int_{\Gamma_{\rho_{2}}\left(z_{0}\right)}|u|^{s}\right)
$$

By the Sobolev inequality, see for instance [1, Chap. 5], we have that

$$
\left(\int_{\Gamma_{\rho_{1}}\left(z_{0}\right)}|u|^{\frac{\hat{n} s}{\hat{n}-2}}\right)^{\frac{\hat{n}-2}{\hat{n} s}} \leqslant\left(\frac{C(1+s)}{\rho_{2}-\rho_{1}}\right)^{\frac{2}{s}}\left(\int_{\Gamma_{\rho_{2}}\left(z_{0}\right)}|u|^{s}\right)^{\frac{1}{s}},
$$

where $\hat{n}=n$ for $n>2, \hat{2}>2$ and $C>0$ is a constant depending on the a priori data only. Now, dealing as in [19, Chap. 8], we observe that the above inequality can be iterated. Indeed, setting $s=s_{m}=2\left(\frac{\hat{n}}{\hat{n}-2}\right)^{m}$ and $\rho_{m}=\frac{r_{0}}{4}+2^{-m} \frac{r_{0}}{4}, m=0,1, \ldots$, by (3.19) it follows

$$
\|u\|_{L^{s_{m}}\left(\Gamma_{\frac{r_{0}}{4}}\left(z_{0}\right)\right)} \leqslant\left(C \frac{\hat{n}}{\hat{n}-2}\right)^{\sum 4 m\left(\frac{\hat{n}}{\hat{n}-2}\right)^{-m}}\|u\|_{L^{2}\left(\Gamma_{\frac{r_{0}}{2}}\left(z_{0}\right)\right)} .
$$

Letting $m$ tends to $\infty$ in (3.19), we can infer that

$$
\|u\|_{L^{\infty}\left(\Gamma_{\frac{r_{0}}{4}}\left(z_{0}\right)\right)} \leqslant C\|u\|_{L^{2}\left(\Gamma_{\frac{r_{0}}{2}}\left(z_{0}\right)\right)},
$$


where $C>0$ is a constant depending on the a priori data only.

Hence combining (3.4) and (3.20) the inequality

$$
\|u\|_{L^{\infty}\left(\Gamma_{\frac{r_{0}}{4}}\left(z_{0}\right)\right)} \leqslant C E,
$$

follows.

Let us now prove the inequality (3.10).

Let $0<r_{1}<r_{2} \leqslant \frac{r_{0}}{4}$ and let us consider a test function $\eta \in \mathrm{C}^{1}(\Omega)$ such that

i) $0 \leqslant \eta \leqslant 1$;

ii) $\eta=1$ in $\Gamma_{r_{1}}\left(z_{0}\right)$ and $\eta=0$ in $\Omega \backslash \Gamma_{r_{2}}\left(z_{0}\right)$;

iii) $|\nabla \eta| \leqslant \frac{2}{r_{2}-r_{1}}$.

By (3.21), we have that

$$
M_{2}=\sup _{x \in \Gamma_{r_{2}}\left(z_{0}\right)} u(x)<+\infty
$$

Let us define the following non-negative function

$$
v(x)=M_{2}-u(x), \text { for every } x \in \Gamma_{r_{2}}\left(z_{0}\right) .
$$

Let us introduce the following quantities.

For every $\rho \in\left(0, \frac{r_{0}}{4}\right)$, let

i) $b=2 L C$,

ii) $h=b M_{2}$;

iii) $k=k(\rho)=\rho^{\delta} h$,

iv) $\bar{b}=b^{2}+k^{-2} h^{2}$;

v) $\bar{v}=v+k$.

where $C>0$ is the constant appearing in the inequality (3.17) and $\delta$ is such that $0<\delta<1$. Let us define, for $\beta \in \mathbb{R} \backslash\{0\}$ the function $\chi=\eta^{2} \bar{v}^{\beta}$. Hence choosing $\chi$ as test function in the weak formulation (3.3), it follows that

$$
\int_{\Gamma_{r_{2}}\left(z_{0}\right)}|\nabla v|^{2} \bar{v}^{\beta-1} \eta^{2}+\frac{2}{\beta} \int_{\Gamma_{r_{2}}\left(z_{0}\right)} \nabla v \cdot \nabla \eta \eta \bar{v}^{\beta}=-\frac{1}{\beta} \int_{\Delta_{r_{2}}\left(z_{0}\right)} f\left(M_{2}-v\right) \eta^{2} \bar{v}^{\beta}
$$

By (3.24), we can infer that

$$
\int_{\Gamma_{r_{2}}\left(z_{0}\right)}|\nabla v|^{2} \bar{v}^{\beta-1} \eta^{2}+\frac{2}{\beta} \int_{\Gamma_{r_{2}}\left(z_{0}\right)} \nabla v \cdot \nabla \eta \eta \bar{v}^{\beta} \leqslant \frac{1}{|\beta|} \int_{\Delta_{r_{2}}\left(z_{0}\right)} L\left|M_{2}-v\right| \eta^{2} \bar{v}^{\beta}
$$


Furthermore by the trace inequality used in (3.17), we have that

$$
\int_{\Gamma_{r_{2}}}|\nabla v|^{2} \bar{v}^{\beta-1} \eta^{2}+\frac{2}{\beta} \int_{\Gamma_{r_{2}}} \nabla v \cdot \nabla \eta \eta \bar{v}^{\beta} \leqslant \frac{L C}{|\beta|} \int_{\Gamma_{r_{2}}\left(z_{0}\right)}\left|\nabla\left[\left(M_{2}-v\right) \eta^{2} \bar{v}^{\beta}\right]\right| .
$$

After straightforward calculations, we have that

$$
\begin{aligned}
& \int_{\Gamma_{r_{2}}\left(z_{0}\right)}|\nabla v|^{2} \bar{v}^{\beta-1} \eta^{2}-L C \int_{\Gamma_{r_{2}}\left(z_{0}\right)}\left|M_{2}-v\right||\nabla v| \bar{v}^{\beta-1} \eta^{2} \leqslant \\
\leqslant & \frac{2}{|\beta|} \int_{\Gamma_{r_{2}}\left(z_{0}\right)}|\nabla v||\nabla \eta| \eta \bar{v}^{\beta}+\frac{2 L C}{|\beta|} \int_{\Gamma_{r_{2}}\left(z_{0}\right)}\left|M_{2}-v\right||\nabla \eta| \eta \bar{v}^{\beta}+ \\
+ & \frac{L C}{|\beta|} \int_{\Gamma_{r_{2}}\left(z_{0}\right)}|\nabla v| \eta^{2} \bar{v}^{\beta} .
\end{aligned}
$$

By the Schwartz inequality it follows that for every $\varepsilon>0$

$$
\begin{aligned}
L C \int_{\Gamma_{r_{2}}\left(z_{0}\right)}\left|M_{2}-v\right||\nabla v| \bar{v}^{\beta-1} \eta^{2} & \leqslant \varepsilon \int_{\Gamma_{r_{2}}\left(z_{0}\right)}|\nabla v|^{2} \bar{v}^{\beta-1} \eta^{2}+ \\
& +\frac{L^{2} C^{2}}{\varepsilon} \int_{\Gamma_{r_{2}}\left(z_{0}\right)}\left|M_{2}-v\right|^{2} \bar{v}^{\beta-1} \eta^{2} .
\end{aligned}
$$

Hence choosing $\varepsilon=\frac{1}{2}$ in (3.27), we obtain

$$
\begin{aligned}
& \int_{\Gamma_{r_{2}}\left(z_{0}\right)}|\nabla v|^{2} \bar{v}^{\beta-1} \eta^{2}-L C \int_{\Gamma_{r_{2}}\left(z_{0}\right)}\left|M_{2}-v \| \nabla v\right| \bar{v}^{\beta-1} \eta^{2} \geqslant \\
\geqslant & \frac{1}{2} \int_{\Gamma_{r_{2}}\left(z_{0}\right)}|\nabla v|^{2} \bar{v}^{\beta-1} \eta^{2}-2 L^{2} C^{2} \int_{\Gamma_{r_{2}}\left(z_{0}\right)}\left|M_{2}-v\right|^{2} \bar{v}^{\beta-1} \eta^{2} \geqslant \\
\geqslant & \frac{1}{2} \int_{\Gamma_{r_{2}}\left(z_{0}\right)}|\nabla v|^{2} \bar{v}^{\beta-1} \eta^{2}-b^{2} \int_{\Gamma_{r_{2}}\left(z_{0}\right)} v^{2} \bar{v}^{\beta-1} \eta^{2}-h^{2} \int_{\Gamma_{r_{2}}\left(z_{0}\right)} \bar{v}^{\beta-1} \eta^{2} .
\end{aligned}
$$

Moreover, observing that $b^{2} v^{2}+h^{2} \leqslant \bar{b} \bar{v}^{2}$, by (3.28) we can infer that

$$
\begin{aligned}
& \int_{\Gamma_{r_{2}}\left(z_{0}\right)}|\nabla v|^{2} \bar{v}^{\beta-1} \eta^{2}-L C \int_{\Gamma_{r_{2}}\left(z_{0}\right)}\left|M_{2}-v\right||\nabla v| \bar{v}^{\beta-1} \eta^{2} \geqslant \\
\geqslant & \frac{1}{2}\left(\int_{\Gamma_{r_{2}}\left(z_{0}\right)}|\nabla v|^{2} \bar{v}^{\beta-1} \eta^{2}-2 \bar{b} \int_{\Gamma_{r_{2}}\left(z_{0}\right)} \bar{v}^{\beta+1} \eta^{2}\right)
\end{aligned}
$$

On the other hand we have also that

$$
\begin{aligned}
& \int_{\Gamma_{r_{2}\left(z_{0}\right)}}|\nabla v||\nabla \eta| \eta \bar{v}^{\beta}+L C \int_{\Gamma_{r_{2}}\left(z_{0}\right)}\left|M_{2}-v\right||\nabla \eta| \eta \bar{v}^{\beta}= \\
\leqslant & \int_{\Gamma_{r_{2}\left(z_{0}\right)}} \frac{1}{2}(2|\nabla v|+b v+h)|\nabla \eta| \eta \bar{v}^{\beta} .
\end{aligned}
$$


Noticing that $b v+h \leqslant 2 \sqrt{b} \bar{v}$, we have that (3.30) yields

$$
\begin{aligned}
& \int_{\Gamma_{r_{2}\left(z_{0}\right)}}|\nabla v||\nabla \eta| \eta \bar{v}^{\beta}+L C \int_{\Gamma_{r_{2}}\left(z_{0}\right)}\left|M_{2}-v\right||\nabla \eta| \eta \bar{v}^{\beta} \leqslant \\
\leqslant & \int_{\Gamma_{r_{2}\left(z_{0}\right)}}(|\nabla v|+\sqrt{\bar{b}} \bar{v})|\nabla \eta| \eta \bar{v}^{\beta} .
\end{aligned}
$$

Hence inserting (3.29) and (3.31) in (3.26) we obtain

$$
\begin{aligned}
& \frac{1}{2}\left(\int_{\Gamma_{r_{2}}\left(z_{0}\right)}|\nabla v|^{2} \bar{v}^{\beta-1} \eta^{2}-2 \bar{b} \int_{\Gamma_{r_{2}}\left(z_{0}\right)} \bar{v}^{\beta+1} \eta^{2}\right) \leqslant \\
\leqslant & \frac{1}{|\beta|} \int_{\Gamma_{r_{2}}\left(z_{0}\right)} \eta \bar{v}^{\beta}|\nabla \eta||\nabla v|+\frac{1}{|\beta|} \int_{\Gamma_{r_{2}}\left(z_{0}\right)} \eta \bar{v}^{\beta+1} \sqrt{\bar{b}}|\nabla \eta|+ \\
+ & \frac{L C}{|\beta|} \int_{\Gamma_{r_{2}}\left(z_{0}\right)}|\nabla v| \eta^{2} \bar{v}^{\beta} .
\end{aligned}
$$

Moreover, by the Schwartz inequality and by (3.32) we obtain that for every $\varepsilon>0$

$$
\begin{aligned}
& \frac{1}{2}\left(\int_{\Gamma_{r_{2}}\left(z_{0}\right)}|\nabla v|^{2} \bar{v}^{\beta-1} \eta^{2}-2 \bar{b} \int_{\Gamma_{r_{2}}\left(z_{0}\right)} \bar{v}^{\beta+1} \eta^{2}\right) \leqslant \\
\leqslant & \frac{\varepsilon}{|\beta|} \int_{\Gamma_{r_{2}}\left(z_{0}\right)}|\nabla v|^{2} \eta^{2} \bar{v}^{\beta-1}+\frac{1}{\varepsilon|\beta|} \int_{\Gamma_{r_{2}\left(z_{0}\right)}}|\nabla \eta|^{2} \bar{v}^{\beta+1}+ \\
+ & \frac{1}{2|\beta|} \int_{\Gamma_{r_{2}}\left(z_{0}\right)}|\nabla \eta|^{2} \bar{v}^{\beta+1}+\frac{\bar{b}}{2|\beta|} \int_{\Gamma_{r_{2}}\left(z_{0}\right)} \eta^{2} \bar{v}^{\beta+1}+ \\
+ & \varepsilon \int_{\Gamma_{r_{2}}\left(z_{0}\right)}|\nabla v|^{2} \bar{v}^{\beta-1} \eta^{2}+\frac{L^{2} C^{2}}{\varepsilon|\beta|^{2}} \int_{\Gamma_{r_{2}}\left(z_{0}\right)} \eta^{2} \bar{v}^{\beta+1} .
\end{aligned}
$$

From the above inequality it follows that

$$
\begin{aligned}
& \left(\frac{1}{2}-\frac{\varepsilon}{|\beta|}-\varepsilon\right) \int_{\Gamma_{r_{2}}\left(z_{0}\right)}|\nabla v|^{2} \bar{v}^{\beta-1} \eta^{2} \leqslant \\
\leqslant & \int_{\Gamma_{r_{2}}\left(z_{0}\right)}\left(2 \bar{b}+\frac{\bar{b}}{2|\beta|}+\frac{L^{2} C^{2}}{\varepsilon|\beta|}\right) \eta^{2} \bar{v}^{\beta+1}+\int_{\Gamma_{r_{2}\left(z_{0}\right)}}\left(\frac{1}{2|\beta|}+\frac{1}{\varepsilon|\beta|}\right)|\nabla \eta|^{2} \bar{v}^{\beta+1} .
\end{aligned}
$$

Thus, choosing $\varepsilon=\min \left\{\frac{1}{8}, \frac{|\beta|}{8}\right\}$, we have that

$$
\int_{\Gamma_{r_{2}}\left(z_{0}\right)}|\nabla v|^{2} \bar{v}^{\beta-1} \eta^{2} \leqslant \hat{C} \int_{\Gamma_{r_{2}}\left(z_{0}\right)}\left(\eta^{2}+|\nabla \eta|^{2}\right) \bar{v}^{\beta+1},
$$

where $\hat{C}$ is a positive constant depending on $|\beta|, L, C, M_{2}, \rho, \delta$. 
Let $w$ be a function defined as follows

$$
w= \begin{cases}\bar{v}^{\frac{\beta+1}{2}}, & \text { if } \beta \neq-1, \\ \log \bar{v}, & \text { if } \beta=-1 .\end{cases}
$$

Hence we can reformulate (3.35) as follows

$$
\int_{\Gamma_{r_{2}}\left(z_{0}\right)}|\eta \nabla w|^{2} \leqslant \begin{cases}(\beta+1)^{2} \hat{C} \int_{\Gamma_{r_{2}}\left(z_{0}\right)}\left[\eta^{2}+|\nabla \eta|^{2}\right] w^{2}, & \text { if } \beta \neq-1, \\ \hat{C} \int_{\Gamma_{r_{2}}\left(z_{0}\right)}\left[\eta^{2}+|\nabla \eta|^{2}\right], & \text { if } \beta=-1 .\end{cases}
$$

By the Sobolev inequality, see for instance [1, Chap. 5], we have that

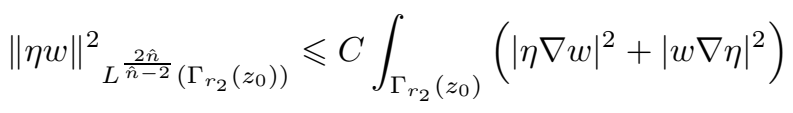

where $\hat{n}=n$ for $n>2, \hat{2}>2$ and $C>0$ is a constant depending on the a priori data only. Combining (3.36) and (3.37) we obtain

$$
\|\eta w\|^{2}{ }_{L^{\frac{2 \hat{n}}{n-2}}\left(\Gamma_{r_{2}}\left(z_{0}\right)\right)} \leqslant c(\beta+1)^{2} \int_{\Gamma_{r_{2}}\left(z_{0}\right)}\left(\eta^{2}+|\nabla \eta|^{2}\right) w^{2},
$$

where $c>0$, depending on the a priori data, on $\rho$, on $|\beta|$ and on $\delta$ only, is bounded when $|\beta|$ is bounded away from zero.

Hence from (3.38) we obtain

$$
\|w\|_{L^{\frac{2 \hat{n}}{\hat{n}-2}}\left(\Gamma_{r_{1}}\left(z_{0}\right)\right)}^{2} \leqslant c^{\prime} \frac{(|\beta+1|+1)}{r_{2}-r_{1}}\|w\|_{L^{2}\left(\Gamma_{r_{2}}\left(z_{0}\right)\right)}
$$

where $c>0$, depending on the a priori data, on $\rho$ and on $\delta$ only.

At this stage arguing as in [19, Theorem 8.18], we obtain the following weak Harnack inequality for the function $v$.

For every $0<\rho<\frac{r_{0}}{16}$, we have that

$$
\rho^{-n}\|v\|_{L^{1}\left(\Gamma_{2 \rho}\left(z_{0}\right)\right)} \leqslant C\left(\inf _{\Gamma_{\rho}\left(z_{0}\right)} v+\rho^{\delta}\left|M_{2}\right|\right)
$$

where $C>0$ is a constant only depending on the a priori data .

On the other hand by (3.21) we have also that,

$$
m_{2}=\inf _{x \in \Gamma_{r_{2}}\left(z_{0}\right)} u(x)<+\infty .
$$

Then, we define the following non-negative function

$$
z(x)=u(x)-m_{2} \text { for every } x \in \Gamma_{r_{2}}\left(z_{0}\right) .
$$


Hence, by analogous arguments to those developed for the function $v$, we find also the following weak Harnack inequality for the function $z$.

For every $0<\rho<\frac{r_{0}}{16}$, we have that

$$
\rho^{-n}\|z\|_{L^{1}\left(\Gamma_{2 \rho}\left(z_{0}\right)\right)} \leqslant C\left(\inf _{\Gamma_{\rho}\left(z_{0}\right)} z+\rho^{\delta}\left|m_{2}\right|\right),
$$

where $C>0$ is a constant only depending on the a priori data .

For every $\rho \in\left(0, \frac{r_{0}}{16}\right)$, let us denote

$$
\begin{array}{r}
M(\rho)=\sup _{\Gamma_{\rho}\left(z_{0}\right)} u, \\
m(\rho)=\inf _{\Gamma_{\rho}\left(z_{0}\right)} u .
\end{array}
$$

By (3.21),(3.40) and (3.43), we have that there exists a constant $K>0$ depending on the $a$ priori data only, such that

$$
\begin{gathered}
\rho^{-n} \int_{\Gamma_{2 \rho}\left(z_{0}\right)}\left(M_{2}-u\right) \leqslant K\left(M_{2}-M+\rho^{\delta}\right), \\
\rho^{-n} \int_{\Gamma_{2 \rho}\left(z_{0}\right)}\left(u-m_{2}\right) \leqslant K\left(m-m_{2}+\rho^{\delta}\right) .
\end{gathered}
$$

Moreover, let us observe that being the boundary $\partial \Omega$ of Lipschitz class, we have that there exists a constant $c_{1}>0$, depending on $r_{0}, M$ only, such that for every $\rho \in\left(0, \frac{r_{0}}{16}\right)$

$$
\rho^{-n}\left|\Gamma_{2 \rho}\left(z_{0}\right)\right| \geqslant c_{1}
$$

Hence adding (3.46) and (3.47), we obtain

$$
M-m \leqslant\left(1-\frac{c_{1}}{K}\right)\left(M_{2}-m_{2}\right)+2 K \rho^{\delta} .
$$

Denoting by $\omega(\rho)=\underset{\Gamma_{\rho}\left(z_{0}\right)}{\operatorname{osc}} u$, we have that by (3.49) it follows

$$
\omega(\rho) \leqslant \gamma \omega(4 \rho)+c_{2} \rho^{\delta},
$$

where $c_{2}=2 K$ and $\gamma=1-\frac{c_{1}}{K}$.

By the arguments in [19, Lemma 8.23], it follows that for any $\mu \in(0,1)$ and any $0<\rho \leqslant$ $\rho_{0} \leqslant \frac{r_{0}}{16}$

$$
\omega(\rho) \leqslant C\left(\left(\frac{\rho}{\rho_{0}}\right)^{\alpha} \omega\left(\rho_{0}\right)+c_{2} \rho^{\mu \delta} \rho_{0}^{(1-\mu) \delta}\right)
$$


where $C$ is a constant depending on the a priori data only, whereas $\alpha$ is such that $\alpha=$ $(1-\mu)\left(\frac{\log (\gamma)}{\log \left(\frac{1}{4}\right)}\right)$. Hence choosing $\mu$ such that $(1-\mu) \frac{\log (\gamma)}{\log \left(\frac{1}{4}\right)}<\mu \delta$, we have that (3.51) leads to

$$
\frac{\omega(\rho)}{\rho^{\alpha}} \leqslant c\left(\rho_{0}^{-\alpha} \omega\left(\rho_{0}\right)+\rho^{\beta}\right)
$$

where $c$ is a constant depending on the a priori data only and $\beta$ is such that $\beta=\mu(\delta-1)-$ $\alpha+1>0$. Furthermore, we have that the above inequality and (3.21) lead to

$$
\frac{\omega(\rho)}{\rho^{\alpha}} \leqslant c\left(\rho_{0}^{-\alpha} 2 C E+\rho^{\beta}\right)
$$

where $C$ is a constant depending on the a priori data only.

Hence we can infer that for any $z_{0} \in \Gamma_{1}$

$$
\|u\|_{C^{0, \alpha}\left(\Gamma_{\frac{r_{0}}{16}}\left(z_{0}\right)\right)} \leqslant C E .
$$

where $C>0,0<\alpha<1$ are constants depending on the a priori data only.

Since, we know that $u \in C^{0, \alpha}\left(\Gamma_{1}\right)$, we have that

$$
\frac{\partial u}{\partial \nu}(x)=-L u(x) \quad \in C^{0, \alpha}\left(\Gamma_{1}\right) .
$$

By well-known regularity bounds for the Neumann problem (see for instance [2, p.667]) it follows that $u \in C^{1, \alpha}\left(U_{\rho}^{1}\right)$ and the following estimate holds

$$
\begin{aligned}
\|u\|_{C^{1, \alpha}\left(U_{\rho}^{1}\right)} & \leqslant C\left(\|u\|_{C^{0, \alpha}\left(\Gamma_{1}^{\frac{\rho}{2}}\right)}+\left\|\frac{\partial u}{\partial \nu}\right\|_{C^{0, \alpha}\left(\Gamma_{1}^{\frac{\rho}{2}}\right)}+\|\nabla u\|_{L^{2}(\Omega)}\right) \leqslant \\
& \leqslant C\left(\left\|\frac{\partial u}{\partial \nu}\right\|_{C^{0, \alpha}\left(\Gamma_{1}^{\frac{\rho}{2}}\right)}+E\right)
\end{aligned}
$$

where $C>0$ depends on the a priori data and on $\rho$ only. Moreover, we can estimate the $C^{0, \alpha}$ norm of $\frac{\partial u}{\partial \nu}$ in terms of $E$, in fact

$$
\begin{aligned}
\left\|\frac{\partial u}{\partial \nu}\right\|_{C^{0, \alpha}\left(\Gamma_{1}^{\frac{\rho}{2}}\right)} & =\sup _{x \in \Gamma_{1}^{\frac{\rho}{2}}}\left|\frac{\partial u(x)}{\partial \nu}\right|+\left(\frac{\rho}{2}\right)^{\alpha} \sup _{x, y \in \Gamma_{1}^{\frac{\rho}{2}}} \frac{\left|\frac{\partial u(x)}{\partial \nu}-\frac{\partial u(y)}{\partial \nu}\right|}{|x-y|^{\alpha}}= \\
& =\sup _{x \in \Gamma_{1}^{\frac{\rho}{2}}}|L u(x)|+L\left(\frac{\rho}{2}\right)^{\alpha} \sup _{x, y \in \Gamma_{1}^{\frac{\rho}{2}}} \frac{|u(x)-u(y)|}{|x-y|^{\alpha}} .
\end{aligned}
$$


Hence we have

$$
\begin{aligned}
\left\|\frac{\partial u}{\partial \nu}\right\|_{C^{0, \alpha}\left(\Gamma_{1}^{\frac{\rho}{2}}\right)} & \leqslant L \sup _{x \in \Gamma_{1}^{\frac{\rho}{2}}}|u(x)|+L\left(\frac{\rho}{2}\right)^{\alpha} \sup _{x, y \in \Gamma_{1}^{\frac{\rho}{2}}} \frac{|u(x)-u(y)|}{|x-y|^{\alpha}} \leqslant \\
& \leqslant L\|u\|_{C^{0, \alpha}\left(\Gamma_{1}\right)} \leqslant C E .
\end{aligned}
$$

So inserting this estimate in (3.55) we obtain the thesis.

Lemma 3.3. The mapping

$$
\begin{aligned}
S_{t}:[0,1] & \rightarrow H^{1}(\Omega) \\
t & \mapsto u_{t}
\end{aligned}
$$

is differentiable and its derivative $u_{t}^{\prime}$ is Lipschitz continuous with respect to t. Moreover, there exists a constant $C>0$, depending on the a priori data only, such that

$$
\begin{aligned}
& \left\|u_{t}^{\prime}\right\|_{H^{1}(\Omega)} \leqslant C\left|L_{1}-L_{0}\right|, \quad \forall t \in[0,1], \\
& \left\|u_{t}^{\prime \prime}\right\|_{H^{1}(\Omega)} \leqslant C\left|L_{1}-L_{0}\right|^{2}, \quad \forall t \in[0,1] .
\end{aligned}
$$

Proof. First we show that the map $S_{t}$ is well defined. Indeed, we observe that since $L_{t}>0$, the well-posedness of the problem (3.2) follows as consequence of the Lax-Milgram theorem.

We show that the mapping $S_{t}$ is Lipschitz continuous.

Let us pick $t_{1}, t_{2} \in[0,1]$, by the weak formulation of the problem (3.2) we have that

$$
\begin{aligned}
\int_{\Omega}\left|\nabla\left(u_{t_{1}}-u_{t_{2}}\right)\right|^{2}= & -\int_{\Gamma_{1}}\left[L_{0}+t_{1}\left(L_{1}-L_{0}\right)\right] u_{t_{1}}\left(u_{t_{1}}-u_{t_{2}}\right) \\
& +\int_{\Gamma_{1}}\left[L_{0}+t_{2}\left(L_{1}-L_{0}\right)\right] u_{t_{2}}\left(u_{t_{1}}-u_{t_{2}}\right) .
\end{aligned}
$$

Hence,

$$
\begin{aligned}
\int_{\Omega}\left|\nabla\left(u_{t_{1}}-u_{t_{2}}\right)\right|^{2}= & \int_{\Gamma_{1}}\left(t_{2}-t_{1}\right)\left(L_{1}-L_{0}\right) u_{t_{1}}\left(u_{t_{1}}-u_{t_{2}}\right) \\
& +\int_{\Gamma_{1}}-\left[L_{0}+t_{2}\left(L_{1}-L_{0}\right)\right]\left(u_{t_{1}}-u_{t_{2}}\right)^{2} .
\end{aligned}
$$

Observing that the second integral on the right hand side is negative, we obtain that

$$
\int_{\Omega}\left|\nabla\left(u_{t_{1}}-u_{t_{2}}\right)\right|^{2} \leqslant \quad \int_{\Gamma_{1}}\left|t_{2}-t_{1}\right|\left|L_{1}-L_{0}\right|\left|u_{t_{1}}\right|\left|u_{t_{1}}-u_{t_{2}}\right| .
$$


Let $\varepsilon>0$, then by the Schwartz inequality and well-known trace inequality (see for instance [1]) we have that

$$
\int_{\Omega}\left|\nabla\left(u_{t_{1}}-u_{t_{2}}\right)\right|^{2} \leqslant \quad \frac{1}{\varepsilon} \int_{\Gamma_{1}}\left|t_{2}-t_{1}\right|^{2}\left|L_{1}-L_{0}\right|^{2}\left|u_{t_{1}}\right|^{2}+C \varepsilon \int_{\Omega}\left|\nabla\left(u_{t_{1}}-u_{t_{2}}\right)\right|^{2},
$$

where $C>0$ is a constant depending on the a priori data only. Hence choosing $\varepsilon=\frac{1}{2 C}$ we obtain that

$$
\int_{\Omega}\left|\nabla\left(u_{t_{1}}-u_{t_{2}}\right)\right|^{2} \leqslant C \int_{\Gamma_{1}}\left|t_{2}-t_{1}\right|^{2}\left|L_{1}-L_{0}\right|^{2}\left|u_{t_{1}}\right|^{2}
$$

where $C>0$ is a constant depending on the a priori data only. Hence by (3.10)

$$
\int_{\Omega}\left|\nabla\left(u_{t_{1}}-u_{t_{2}}\right)\right|^{2} \leqslant C \int_{\Gamma_{1}}\left|t_{2}-t_{1}\right|^{2}\left|L_{1}-L_{0}\right|^{2}
$$

where $C>0$ is a constant depending on the a priori data only. By (3.67) we deduce that the map $S_{t}$ is Lipschitz continuous and therefore, for almost every $t$, the derivative $u_{t}^{\prime}$ exists and satisfies

$$
\left\{\begin{array}{lc}
\Delta u_{t}^{\prime}=0, & \text { in } \Omega, \\
\frac{\partial u_{t}^{\prime}}{\partial \nu}=0, & \text { on } \Gamma_{2}, \\
\frac{\partial u_{t}^{\prime}}{\partial \nu}+L_{t} u_{t}^{\prime}=-\left(L_{1}-L_{0}\right) u_{t}, & \text { on } \Gamma_{1}, \\
u_{t}^{\prime}=0, & \text { on } \Gamma_{D} .
\end{array}\right.
$$

Moreover by (3.67), (3.59) follows for almost every $t$.

By analogous arguments we can prove that $u_{t}^{\prime}$ is Lipschitz continuous. Therefore, (3.59) holds for every $t$ and, for almost every $t$, the second derivative $u_{t}^{\prime \prime}$ satisfies

$$
\left\{\begin{array}{lc}
\Delta u_{t}^{\prime \prime}=0, & \text { in } \Omega, \\
\frac{\partial u_{t}^{\prime \prime}}{\partial \nu^{\prime \prime}}=0, & \text { on } \Gamma_{2}, \\
\frac{\partial u_{t}^{\prime \prime}}{\partial \nu}+L_{t} u_{t}^{\prime \prime}=-2\left(L_{1}-L_{0}\right) u_{t}^{\prime}, & \text { on } \Gamma_{1}, \\
u_{t}^{\prime \prime}=0, & \text { on } \Gamma_{D} .
\end{array}\right.
$$

We have that (3.60) follows for almost every $t \in[0,1]$ dealing with similar argument to those used to prove (3.59). Moreover, arguing as before, we observe that the map $t \rightarrow u_{t}^{\prime \prime}$ is Lipschitz continuous and we can conclude that (3.60) holds for every $t \in[0,1]$.

Theorem 3.4. There exists a constant $C>0$, depending on the a priori data only, such that

$$
\begin{aligned}
& \left\|u_{t}^{\prime}\right\|_{L^{2}\left(\Gamma_{2}\right)} \leqslant C\left|L_{1}-L_{0}\right|, \quad \forall t \in[0,1], \\
& \left\|u_{t}^{\prime \prime}\right\|_{L^{2}\left(\Gamma_{2}\right)} \leqslant C\left|L_{1}-L_{0}\right|^{2}, \quad \forall t \in[0,1] .
\end{aligned}
$$


Proof. By a standard trace inequality (see for instance [1]) and by the previous Lemma, we deduce that

$$
\begin{aligned}
& \left\|u_{t}^{\prime}\right\|_{L^{2}\left(\Gamma_{2}\right)} \leqslant C\left|L_{1}-L_{0}\right|, \quad \forall t \in[0,1] \\
& \left\|u_{t}^{\prime \prime}\right\|_{L^{2}\left(\Gamma_{2}\right)} \leqslant C\left|L_{1}-L_{0}\right|^{2}, \quad \forall t \in[0,1] .
\end{aligned}
$$

By the Taylor formula we have that

$$
u_{1}-u_{0}=u_{0}^{\prime}+R \text { on } \Gamma_{2}
$$

where $R=\int_{0}^{1}(t-1) u_{t}^{\prime \prime} \mathrm{d} t$ satisfies

$$
\|R\|_{L^{2}\left(\Gamma_{2}\right)} \leqslant C\left|L_{0}-L_{1}\right|^{2} .
$$

Lemma 3.5. There exists a constant $K>0$ depending on the a priori data only such that

$$
\left\|u_{0}\right\|_{L^{\infty}\left(\Gamma_{1}\right)} \geqslant K .
$$

Proof. Dealing as in [7, Theorem 2.1] we obtain that

$$
\left\|u_{0}\right\|_{L^{\infty}\left(\Gamma_{1}\right)} \geqslant \eta\left(\|g\|_{L^{\infty}\left(\Gamma_{2,2 r_{0}}\right)}\right) \geqslant 0
$$

where $\eta$ is the function defined in the formula (2.15) in [7]. Hence the thesis follows.

Lemma 3.6. Let $u_{0}^{\prime}$ be the solution to (3.68) with $t=0$, then for any $\rho \in\left(0, r_{0}\right) u_{0}^{\prime} \in$ $C^{1, \alpha}\left(U_{\rho}^{1}\right)$ and there exists a constant $C_{\rho}>0$, depending on the a priori data and on $\rho$ only, such that the following estimate holds

$$
\left\|u_{0}^{\prime}\right\|_{C^{1, \alpha}\left(U_{\rho}^{1}\right)} \leqslant C_{\rho}
$$

where $\rho \in\left(0, r_{0}\right)$ and $0<\alpha<1$ is a constant depending on $r_{0}, M, n$ only.

Proof. First we shall prove an upper bound for the energy of $u_{0}^{\prime}$. Indeed, by the weak formulation of (3.68) we have that

$$
\int_{\Omega}\left|\nabla u_{0}^{\prime}\right|^{2}+\int_{\Gamma_{1}} L_{0}\left|u_{0}^{\prime}\right|^{2}=\int_{\Gamma_{1}}\left(L_{0}-L_{1}\right) u_{0} u_{0}^{\prime} .
$$

Let $\varepsilon>0$, applying the Scwhartz inequality the right hand side we have

$$
\int_{\Omega}\left|\nabla u_{0}^{\prime}\right|^{2}+\int_{\Gamma_{1}} L_{0}\left|u_{0}^{\prime}\right|^{2} \leqslant \varepsilon \int_{\Gamma_{1}}\left|u_{0}^{\prime}\right|^{2}+\frac{1}{\varepsilon} \int_{\Gamma_{1}}\left|L_{0}-L_{1}\right| u_{0}^{2}
$$

$\mathrm{RR} \mathrm{n}^{\circ} 6103$ 
Moreover, observing that the second term on the left hand side is positive we have that

$$
\int_{\Omega}\left|\nabla u_{0}^{\prime}\right|^{2} \leqslant \varepsilon \int_{\Gamma_{1}}\left|u_{0}^{\prime}\right|^{2}+\frac{2}{\varepsilon} \Lambda E .
$$

Choosing $\varepsilon>0$ sufficiently small and by a trace inequality we have that, there exists a constant $C>0$ depending on the a priori data only, such that

$$
\int_{\Omega}\left|\nabla u_{0}^{\prime}\right|^{2} \leqslant C
$$

By Theorem 3.2 we know that $u_{0} \in C^{0, \alpha}\left(\Gamma_{1}\right)$. Hence by well-known regularity results for the Neumann problem and using the same tecniques used in Theorem (3.2) as well as in [24, Chap. 3] we deduce the thesis.

Proposition 3.7. There exists a constant $C>0$ depending on the a priori data only, such that

$$
\left\|u_{0}^{\prime}\right\|_{L^{2}\left(\Gamma_{2}\right)} \geqslant c\left|L_{0}-L_{1}\right| .
$$

Proof. Let us define the function

$$
\tilde{u_{0}}=\frac{u_{0}^{\prime}}{L_{0}-L_{1}}
$$

where $u_{0}^{\prime}$ is the solution to the problem (3.69) with $t=0$.

It follows that $\tilde{u_{0}}$ is the solution to the following problem

$$
\begin{cases}\Delta \tilde{u_{0}}=0, & \text { in } \Omega, \\ \frac{\partial \tilde{u_{0}}}{\partial \nu}=0, & \text { on } \Gamma_{2}, \\ \frac{\partial \tilde{u_{0}}}{\partial \nu}+L_{0} \tilde{u_{0}}=u_{0}, & \text { on } \Gamma_{1}, \\ \tilde{u_{0}}=0, & \text { on } \Gamma_{D}\end{cases}
$$

Elaborating a classical estimate of unique continuation from the boundary [25], see also [24][Chap.2] we have that

$$
\left\|\tilde{u_{0}}\right\|_{L^{2}\left(B_{\rho}\left(P_{0}\right) \cap U_{2 r_{0}}^{2}\right)} \leqslant c\left(\left\|\tilde{u_{0}}\right\|_{L^{2}\left(\Gamma_{2}\right)}+\left\|\nabla \tilde{u_{0}}\right\|_{H^{1}(\Omega)}\right)^{1-\delta}\left\|\tilde{u_{0}}\right\|_{L^{2}\left(\Gamma_{2}\right)}^{\delta},
$$

where $\rho \in\left(\frac{M}{4 \sqrt{1+M^{2}} r_{0}}, \frac{3 M}{4 \sqrt{1+M^{2}} r_{0}}\right), P_{0}=P_{1}+\frac{M}{4 \sqrt{1+M^{2}} r_{0}} \nu, \nu$ is outer unit normal to $\Omega$ at $P_{1}$ and $c>0,0<\delta<1$ are constants depending on the a priori data and on $\rho$ only. By (3.59) we have that

$$
\left\|\tilde{u_{0}}\right\|_{H^{1}(\Omega)} \leqslant C .
$$


Hence we have that

$$
\left\|\tilde{u_{0}}\right\|_{L^{2}\left(B_{\rho}\left(P_{0}\right) \cap U_{2 r_{0}}^{2}\right)} \leqslant c\left(\left\|\tilde{u_{0}}\right\|_{L^{2}\left(\Gamma_{2}\right)}+C\right)^{1-\delta}\left\|\tilde{u_{0}}\right\|_{L^{2}\left(\Gamma_{2}\right)}^{\delta}
$$

where $C>0$ is the constant introduced in (3.59).

By the Lipschitz regularity of the boundary we have that if $Q$ is a point of $\partial \Omega$, then there exists a rigid transformation of coordinates under which we have $Q=0$. We consider the finite cone

$$
\mathcal{C}=\left\{x:|x|<r_{0}, \frac{x \cdot \xi}{|x|}>\cos \theta\right\}
$$

with axis in the direction $\xi$ and width $2 \theta$, where $\theta=\arctan \frac{1}{M}$, we have that $\mathcal{C} \subset \Omega$. Let us consider now a point $Q \in \Gamma_{2, r_{0}}$ and let $Q_{0}$ be a point lying on the axis $\xi$ of the cone with vertex in $Q=0$ such that $d_{0}=\operatorname{dist}\left(Q_{0}, 0\right)<\frac{r_{0}}{2}$. Following Lieberman [22], we introduce a regularized distance $\tilde{d}$ from the boundary of $\Omega$. We have that there exists $\tilde{d}$ such that $\tilde{d} \in C^{2}(\Omega) \cap C^{0,1}(\bar{\Omega})$, satisfying the following properties

- $\gamma_{0} \leqslant \frac{\operatorname{dist}(x, \partial \Omega)}{\tilde{d}(x)} \leqslant \gamma_{1}$,

- $|\nabla \tilde{d}(x)| \geqslant c_{1}, \quad$ for every $x$ such that $\operatorname{dist}(x, \partial \Omega) \leqslant b r_{0}$,

- $\|\tilde{d}\|_{C^{1, \alpha}} \leqslant c_{2} r_{0}$,

where $\gamma_{0}, \gamma_{1}, c_{1}, c_{2}, b$ are positive constants depending on $M, \alpha$ only, (see also [3, Lemma $5.2])$.

Let us define for every $\rho>0$

$$
\tilde{\Omega}_{\rho}=\{x \in \Omega \quad: \quad \tilde{d}(x)>\rho\} .
$$

We have that, there exists $a, 0<a \leqslant 1$, only depending on $M, \alpha$ such that for every $\rho$, $0<\rho \leqslant a r_{0}, \tilde{\Omega}_{\rho}$ is connected with boundary of class $C^{1}$ and

$$
\tilde{c}_{1} \rho \leqslant \operatorname{dist}(x, \partial \Omega) \leqslant \tilde{c}_{2} \rho \quad \text { for every } x \in \partial \tilde{\Omega}_{\rho} \cap \Omega
$$

where $\tilde{c}_{1}, \tilde{c}_{2}$ are positive constants depending on $M, \alpha$ only. By (3.89) it follows that

$$
\Omega_{\tilde{c}_{2} \rho} \subset \tilde{\Omega}_{\rho} \subset \Omega_{\tilde{c}_{1} \rho} .
$$

We define the point $P=P_{0}-\frac{1}{4 \sqrt{1+M^{2}}} r_{0} \cdot \nu$ and $\rho_{0}=\min \left\{\frac{1}{32 M \sqrt{1+M^{2}}} r_{0}, \frac{r_{0}}{4} \sin \theta\right\}$. Moreover, let $\gamma$ be a path in $\tilde{\Omega}_{\frac{\rho_{0}}{\bar{c}_{1}}}$ joining $P$ to $Q_{0}$ and let us define $\left\{y_{i}\right\}, i=0, \ldots, s$ as follows $y_{0}=Q_{0}$, $y_{i+1}=\gamma\left(t_{i}\right)$, where $t_{i}=\max \left\{t\right.$ s.t. $\left.\left|\gamma(t)-y_{i}\right|=2 \rho_{0}\right\}$ if $\left|P-y_{i}\right|>2 \rho_{0}$ otherwise let $i=s$ and stop the process. 
Now, we will use the three spheres inequality for harmonic functions (see for instance [21] or $[6$, Appendix E]) that is

$$
\int_{B_{3 \rho_{0}}\left(y_{0}\right)}{\tilde{u_{0}}}^{2} \leqslant\left(\int_{B_{\rho_{0}}\left(y_{0}\right)}{\tilde{u_{0}}}^{2}\right)^{\tau} \cdot\left(\int_{B_{4 \rho_{0}}\left(y_{0}\right)}{\tilde{u_{0}}}^{2}\right)^{1-\tau}
$$

where $0<\tau<1$ is an absolute constant. Now since $B_{\rho_{0}}\left(y_{0}\right) \subset B_{3 \rho_{0}}\left(y_{1}\right)$ and since by $(3.87)$ $\left\|\tilde{u_{0}}\right\|_{H^{1}(\Omega)} \leqslant C$, then we have

$$
\int_{B_{\rho_{0}}\left(y_{0}\right)}{\tilde{u_{0}}}^{2} \leqslant\left(\int_{B_{3 \rho_{0}}\left(y_{1}\right)}{\tilde{u_{0}}}^{2}\right)^{\tau} \cdot C^{1-\tau} .
$$

An iterated application of the three spheres inequality leads to

$$
\int_{B_{\rho_{0}}\left(y_{0}\right)}{\tilde{u_{0}}}^{2} \leqslant\left(\int_{B_{\rho_{0}}\left(y_{s}\right)}{\tilde{u_{0}}}^{2}\right)^{\tau^{s}} \cdot C^{1-\tau^{s}} .
$$

Finally, since we have $B_{\rho_{0}}\left(y_{s}\right) \subset B \frac{3 M}{4 \sqrt{1+M^{2}}} r_{0}\left(P_{0}\right) \cap U_{2 r_{0}}^{2}$, then by the Proposition (3.88) it follows

$$
\int_{B_{\rho_{0}}\left(y_{0}\right)}{\tilde{u_{0}}}^{2} \leqslant C\left\{(\varepsilon+C)^{1-\delta} \cdot\left(\left\|\tilde{u_{0}}\right\|_{L^{2}\left(\Gamma_{2}\right)}\right)^{\delta}\right\}^{\tau^{s}}
$$

We shall construct a chain of balls $B_{\rho_{k}}\left(Q_{k}\right)$ centered on the axis of the cone, pairwise tangent to each other and all contained in the cone

$$
\mathcal{C}^{\prime}=\left\{x:|x|<r_{0}, \frac{x \cdot \xi}{|x|}>\cos \theta^{\prime}\right\}
$$

where $\theta^{\prime}=\arcsin \left(\frac{\rho_{0}}{d_{0}}\right)$. Let $B_{\rho_{0}}\left(Q_{0}\right)$ be the first of them, the following are defined by induction in such a way

$$
\begin{aligned}
& Q_{k+1}=Q_{k}-(1+\mu) \rho_{k} \xi \\
& \rho_{k+1}=\mu \rho_{k}, \\
& d_{k+1}=\mu d_{k},
\end{aligned}
$$

with

$$
\mu=\frac{1-\sin \theta^{\prime}}{1+\sin \theta^{\prime}} .
$$

Hence, with this choice, we have $\rho_{k}=\mu^{k} \rho_{0}$ and $B_{\rho_{k+1}}\left(Q_{k+1}\right) \subset B_{3 \rho_{k}}\left(Q_{k}\right)$.

Let us now consider the following estimate obtained by a repeated application of the three 
spheres inequality

$$
\begin{aligned}
\left\|\tilde{u_{0}}\right\|_{L^{2}\left(B_{\rho_{k}}\left(Q_{k}\right)\right)} & \leqslant\left\|\tilde{u_{0}}\right\|_{L^{2}\left(B_{3 \rho_{k-1}}\left(Q_{k-1}\right)\right)} \leqslant \\
& \leqslant\left\|\tilde{u_{0}}\right\|_{L^{2}\left(B_{\rho_{k-1}}\left(Q_{k-1}\right)\right)}^{\tau}\left\|\tilde{u_{0}}\right\|_{L^{2}\left(B_{4 \rho_{l-1}}\left(Q_{k-1}\right)\right)}^{1-\tau} \\
& \leqslant C\left\|\tilde{u_{0}}\right\|_{L^{2}\left(B_{\rho_{0}}\left(Q_{0}\right)\right)}^{\tau^{k}} \leqslant \\
& \leqslant C\left\{\left[\left(\left\|\tilde{u_{0}}\right\|_{L^{2}\left(\Gamma_{2}\right)}+C\right)^{1-\delta} \cdot\left(\left\|\tilde{u_{0}}\right\|_{L^{2}\left(\Gamma_{2}\right)}\right)^{\delta}\right]^{\tau^{s}}\right\}^{\tau^{k}} .
\end{aligned}
$$

For every $r, 0<r<d_{0}$, let $k(r)$ be the smallest positive integer such that $d_{k} \leqslant r$, then since $d_{k}=\mu^{k} d_{0}$, it follows

$$
\frac{\left|\log \left(\frac{r}{d_{0}}\right)\right|}{\log \mu} \leqslant k(r) \leqslant \frac{\left|\log \left(\frac{r}{d_{0}}\right)\right|}{\log \mu}+1
$$

and by (3.90), we have

$$
\left\|\tilde{u_{0}}\right\|_{L^{2}\left(B_{\rho_{k}(r)}\left(Q_{k}(r)\right)\right)} \leqslant C\left\{\left[\left(\left\|\tilde{u_{0}}\right\|_{L^{2}\left(\Gamma_{2}\right)}+C\right)^{1-\delta} \cdot\left(\left\|\tilde{u_{0}}\right\|_{L^{2}\left(\Gamma_{2}\right)}\right)^{\delta}\right]^{\tau^{s}}\right\}^{\tau^{k(r)}} .
$$

Let $\bar{x} \in \Gamma_{1,2 r_{0}}, x \in B \frac{\rho_{k(r)-1}}{2}\left(Q_{k(r)-1}\right)$, since $\tilde{u}_{0} \in C^{1, \alpha}\left(U_{2 r_{0}}^{1}\right)$ we have

$$
\left|\frac{\partial \tilde{u}_{0}(\bar{x})}{\partial \nu}\right| \leqslant\left|\frac{\partial \tilde{u_{0}}(x)}{\partial \nu}\right|+C|x-\bar{x}|^{\alpha} .
$$

Hence by (3.85) we have that

$$
\left|u_{0}(\bar{x})\right| \leqslant\left|L_{0} \tilde{u_{0}}(\bar{x})\right|+\left|\frac{\partial \tilde{u_{0}}(x)}{\partial \nu}\right|+C|x-\bar{x}|^{\alpha} .
$$

Moreover, by the Hölder continuity of $\tilde{u_{0}}$ we have that

$$
\left|u_{0}(\bar{x})\right| \leqslant\left|L_{0} \tilde{u_{0}}(x)\right|+\left|\frac{\partial \tilde{u_{0}}(x)}{\partial \nu}\right|+C\left(1+L_{0}\right)|x-\bar{x}|^{\alpha} .
$$

Hence we have

$$
\left|u_{0}(\bar{x})\right| \leqslant\left|L_{0} \tilde{u_{0}}(x)\right|+\left|\frac{\partial \tilde{u_{0}}(x)}{\partial \nu}\right|+C\left(\frac{2 r}{\mu}\right)^{\alpha},
$$

where $C>0$ is a constant depending on the a priori data only.

Moreover, by the estimate (3.76) we have that

$$
K \leqslant\left|L_{0} \tilde{u_{0}}(x)\right|+\left|\frac{\partial \tilde{u_{0}}(x)}{\partial \nu}\right|+C\left(\frac{2 r}{\mu}\right)^{\alpha} .
$$


Integrating over $\frac{\beta_{k_{(r)-1}}}{2}\left(Q_{k(r)-1}\right)$, we deduce that

$$
\begin{aligned}
& K^{2} \leqslant \frac{2 L_{0}}{\omega_{n}\left(\frac{\rho_{k-1}}{2}\right)^{n}} \int_{\frac{\rho_{k(r)-1}}{2}\left(Q_{k(r)-1}\right)}\left|\tilde{u_{0}}(x)\right|^{2} \mathrm{~d} x+ \\
& +\frac{2}{\omega_{n}\left(\frac{\rho_{k-1}}{2}\right)^{n}} \int_{\frac{\rho_{\frac{\rho_{k(r)-1}}{2}}}{2}\left(Q_{k(r)-1}\right)}\left|\frac{\partial \tilde{u_{0}}(x)}{\partial \nu}\right|^{2} \mathrm{~d} x+2 C^{2}\left(\frac{4 r^{2}}{\mu^{2}}\right)^{\alpha} \leqslant \\
& \leqslant \frac{2 L_{0}}{\omega_{n}\left(\frac{\rho_{k-1}}{2}\right)^{n}} \int_{B_{\frac{\rho_{k(r)-1}}{2}}\left(Q_{k(r)-1}\right)}\left|\tilde{u_{0}}(x)\right|^{2} \mathrm{~d} x+ \\
& +\frac{2}{\omega_{n}\left(\frac{\rho_{k-1}}{2}\right)^{n}} \int_{\frac{\rho_{\frac{\rho_{k(r)-1}}{2}}}{2}\left(Q_{k(r)-1}\right)}\left|\nabla \tilde{u_{0}}(x)\right|^{2} \mathrm{~d} x+2 C^{2}\left(\frac{4 r^{2}}{\mu^{2}}\right)^{\alpha} .
\end{aligned}
$$

Applying the Caccioppoli inequality, we have

$$
\left|\frac{\partial u(\bar{x})}{\partial \nu}\right|^{2} \leqslant \frac{C}{\left(\rho_{k-1}\right)^{n+2}} \int_{B_{\rho_{k(r)-1}}\left(Q_{k(r)-1}\right)}\left|\tilde{u}_{0}(x)\right|^{2} \mathrm{~d} x+C r^{2 \alpha}
$$

and since $k$ is the smallest integer such that $d_{k} \leqslant r$, then $d_{k-1}>r$, it follows

$$
K^{2} \leqslant \frac{C}{\left(r \sin \theta^{\prime}\right)^{n+2}} \int_{B_{\rho_{k(r)-1}}\left(Q_{k(r)-1}\right)}\left|\tilde{u}_{0}(x)\right|^{2} \mathrm{~d} x+C r^{2 \alpha} .
$$

From (3.92), we deduce

$$
K^{2} \leqslant \frac{C}{r^{n+2}}\left\{\left[\left(\left\|\tilde{u_{0}}\right\|_{L^{2}\left(\Gamma_{2}\right)}+C\right)^{1-\delta} \cdot\left(\left\|\tilde{u_{0}}\right\|_{L^{2}\left(\Gamma_{2}\right)}\right)^{\delta}\right]^{\tau^{s}}\right\}^{\tau^{k(r)-1}}+C r^{2 \alpha} .
$$

Let us define

$$
\sigma\left(\left\|\tilde{u_{0}}\right\|_{L^{2}\left(\Gamma_{2}\right)}\right)=\left[\left(\left\|\tilde{u_{0}}\right\|_{L^{2}\left(\Gamma_{2}\right)}+C\right)^{1-\delta} \cdot\left(\left\|\tilde{u_{0}}\right\|_{L^{2}\left(\Gamma_{2}\right)}\right)^{\delta}\right]^{\tau^{s}},
$$

thus the previous inequality becomes

$$
K^{2} \leqslant \frac{C}{r^{n+2}}\left\{\sigma\left(\left\|\tilde{u_{0}}\right\|_{L^{2}\left(\Gamma_{2}\right)}\right)\right\}^{\tau^{k(r)-1}}+C r^{2 \alpha} .
$$

Now, using (3.91), we have

$$
\tau^{k(r)-1} \geqslant\left(\frac{r}{d_{0}}\right)^{\nu}
$$

where $\nu=-\log \left(\frac{1}{\mu}\right) \log \tau$. We have

$$
K \leqslant C\left\{r^{-\frac{n+2}{2}}\left[\sigma\left(\left\|\tilde{u_{0}}\right\|_{L^{2}\left(\Gamma_{2}\right)}\right)\right]^{\frac{r^{\nu}}{2}}+r^{\alpha}\right\} .
$$

INRIA 
Now minimizing the function on the right hand side, with respect to $r$, with $r \in\left(0, \frac{r_{0}}{4}\right)$, we deduce

$$
K \leqslant C\left(\log \frac{1}{\sigma\left(\left\|\tilde{u_{0}}\right\|_{L^{2}\left(\Gamma_{2}\right)}\right)}\right)^{-\frac{2 \alpha}{\nu+2}} .
$$

Hence, solving for $\left\|\tilde{u_{0}}\right\|_{L^{2}\left(\Gamma_{2}\right)}$, we can compute

$$
\left\|\tilde{u_{0}}\right\|_{L^{2}\left(\Gamma_{2}\right)} \geqslant C \exp \left\{-K^{-\frac{\nu+2}{2 \alpha}}\right\} .
$$

By the definition of $\tilde{u_{0}}$ we obtain the thesis.

Lemma 3.8. There exists a continuous, increasing function $\omega:[0,+\infty) \rightarrow[0,+\infty)$, with $\omega(0)=0$, such that

$$
\left|L_{0}-L_{1}\right| \leqslant \omega\left(\left\|u_{0}-u_{1}\right\|_{L^{2}\left(\Gamma_{2}\right)}\right)
$$

Proof. For the proof we refer to [7, Theorem 2.2], see also [24].

Proof. Theorem 2.1. If $\left|L_{0}-L_{1}\right| \leqslant \frac{c}{2}$ where $c>0$ is the constant in (3.83), then from (3.74)-(3.83), we deduce

$$
\left\|u_{1}-u_{0}\right\|_{L^{2}\left(\Gamma_{2}\right)} \geqslant\left|L_{1}-L_{0}\right|\left(c-C\left|L_{0}-L_{1}\right|\right) \geqslant \frac{c}{2}\left|L_{0}-L_{1}\right|
$$

Then (2.8) follows.

On the other hand if $\left|L_{0}-L_{1}\right| \leqslant \frac{c}{2}$, we have that by

$$
\left|L_{1}-L_{0}\right| \leqslant \frac{2 \Lambda}{\omega^{-1}\left(\frac{c}{2 C}\right)}\left\|u_{0}-u_{1}\right\|_{L^{2}\left(\Gamma_{2}\right)},
$$

thus (2.8) follows as well.

\section{References}

[1] R.A. Adams, Sobolev Spaces, Academic Press, New York, 1975.

[2] S. Agmon, A. Douglis, L. Niremberg, Estimates Near the Boundary for Solution of Elliptic Partial Differential Equationns Satisfying General Boundary Conditions. I., Comm. Pure Appl. Math., 12, (1959), 623-727.

[3] G. Alessandrini, E. Beretta, E. Rosset, S. Vessella, Optimal Stability for Inverse Elliptic Boundary Value Problems with Unknown Boundaries, Ann. Sc. Norm. Super. Pisa Scienze Fisiche e Matematiche - Serie IV. Vol XXIX. Fasc.4 (2000). 
[4] G. Alessandrini, E. Beretta, S. Vessella, Determining linear cracks by boundary measurements: Lipschitz stability, SIAM J. Math. Anal. 27 (2) (1996) 361-375.

[5] G. Alessandrini, L. Del Piero, L. Rondi, Stable determination of corrosion by a single electrostatic boundary measurement, Inverse Problem, 19 (2003), no. 4, 973-984.

[6] G. Alessandrini, E. DiBenedetto, Determining 2-dimensional cracks in 3-dimensional bodies: uniqueness and stability, Indiana Univ. Math. J., 46 (1997), no 1, 1-82.

[7] G. Alessandrini, E. Sincich, Detecting nonlinear corrosion by electrostatic measurements, Applicable Anal. 85, 1-3 January-March 2006, 107-128.

[8] G. Alessandrini, E. Sincich, Solving elliptic Cauchy problems and the identification of nonlinear corrosion, to appear on J. Comput. Appl. Math.

[9] G. Alessandrini, S. Vessella, Lipschitz Stability for the Inverse Conductivity Problem, Adv. Appl. Math. 35, 2 (August 2005) 207-241.

[10] V. Bacchelli, S. Vessella, Lipschitz stability for a stationary 2-D inverse problem with unknown poligonal boundary, Inverse Problems 22, No.5 (October 2006), 1627-1658.

[11] H. Bellout, A. Friedman, Identification problems in potential theory, Arch. Ration. Mech. Anal. 101, 1988, 143-160.

[12] K. Bryan, M. Vogelius, Singular solutions to a nonlinear elliptic boundary value problem originating from corrosion modeling, Quart. Appl. Math., Vol.60, (2002), 675-694.

[13] S. Chaabane, I. Fellah, M. Jaoua, J. Leblond, Logarithmic stability estimates for a Robin coefficient in two-dimensional Laplace inverse oroblems, Inverse Problems, 20 (2004), no.1, 47-59.

[14] S. Chaabane, M. Jaoua, J. Leblond, Parameter identification for the Laplace equation and approximation in Hardy classes, J. Inverse Ill-Posed Proble. 11 (2003), no.1, 33-57.

[15] S. Chaabane, M. Jaoua, Identification of Robin coefficients by the means of boundary measurements, Inverse Problems 15 (1999), no. 6, 1425-1438.

[16] D. Fasino, G. Inglese, An inverse Robin problem for Laplace s equation: theoretical results and numerical methods, Inverse Problems, 15, 41-48, (1999).

[17] D. Fasino, G. Inglese, Discrete methods in the study of an inverse problem for Laplace's equation, IMA J. Numer. Anal., 19, no.1, 105-118, (1999).

[18] D. Fasino, G. Inglese, Stability of the solutions of an inverse problem for Laplace's equation in a thin strip, Numer. Funct. Anal. Optim. 22 (2001), no.5-6, 549-560.

[19] D. Gilbarg, N.S. Trudinger, Elliptic Partial Differential Equations od Second Order, Second edition, Springer-Verlag, Heidelberg, (1977). 
[20] O. Kavian, M. Vogelius, On the existence and "blow-up" of solutions of a two dimensional nonlinear boundary value problem arising in corrosion modeling, Proc. Roy. Soc. Edinburgh, Vol.133A, 119-149, (2003).

[21] J. Korevaar, J.L.H. Meyers, Logarithmic convexity for supremum norms of harmonic functions, Bull. London ath. Soc., 26 (1994), no.4, 335-362.

[22] G.M. Lieberman, Regularized distance and its applications, Pacific J. Math. 117, (1985), 329-353.

[23] J.L. Lions, E. Magenes, Non-Homogeneous Boundary Value Problems and Applications, Vol.1, Springer-Verlag, 1972.

[24] E. Sincich, Stability and Reconstruction for the Determination of Boundary Terms by a Single Measurements, PhD Thesis, SISSA-ISAS, Trieste, 2005. (down-loadable at http:// www.sissa.it/library/).

[25] G.N. Trytten, Pointwise bound for solution of the Cauchy problem for elliptic equations, Arch. Ration. Mech. Anal. 13 (1963), 222-224.

[26] M.Vogelius, J.-M. Xu, A nonlinear elliptic boundary value problem related to corrosion modeling, Quart. Appl. Math., Vol.56, 479-505, (1998). 


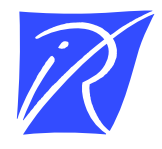

\section{Unité de recherche INRIA Sophia Antipolis 2004, route des Lucioles - BP 93 - 06902 Sophia Antipolis Cedex (France)}

Unité de recherche INRIA Futurs : Parc Club Orsay Université - ZAC des Vignes 4, rue Jacques Monod - 91893 ORSAY Cedex (France)

Unité de recherche INRIA Lorraine : LORIA, Technopôle de Nancy-Brabois - Campus scientifique 615, rue du Jardin Botanique - BP 101 - 54602 Villers-lès-Nancy Cedex (France)

Unité de recherche INRIA Rennes : IRISA, Campus universitaire de Beaulieu - 35042 Rennes Cedex (France)

Unité de recherche INRIA Rhône-Alpes : 655, avenue de l'Europe - 38334 Montbonnot Saint-Ismier (France)

Unité de recherche INRIA Rocquencourt : Domaine de Voluceau - Rocquencourt - BP 105 - 78153 Le Chesnay Cedex (France)

INRIA - Domaine de Voluceau - Rocquencourt, BP 105 - 78153 Le Chesnay Cedex (France)

http://www.inria.fr

ISSN 0249-6399 